Témoigner Témoigner. Entre histoire et mémoire

Getuigen Revue pluridisciplinaire de la Fondation Auschwitz

$118 \mid 2014$

Au nom des victimes. Dictature et terreur d'État en Argentine, Chili et Uruguay

\title{
Le musée juif et le centre pour la tolérance de Moscou
}

The Jewish Museum and Tolerance Centre in Moscow

Het Joodse Museum en Centrum voor Tolerantie in Moskou

\section{Ewa Bérard}

\section{(2) OpenEdition}

Journals

\section{Édition électronique}

URL : http://journals.openedition.org/temoigner/1224

DOI : 10.4000/temoigner.1224

ISSN : 2506-6390

Éditeur :

Éditions du Centre d'études et de documentation Mémoire d'Auschwitz, Éditions Kimé

Édition imprimée

Date de publication : 1 octobre 2014

Pagination : 14-17

ISBN : 978-2-84174-674-3

ISSN : 2031-4183

Référence électronique

Ewa Bérard, "Le musée juif et le centre pour la tolérance de Moscou », Témoigner. Entre histoire et mémoire [En ligne], 118 | 2014, mis en ligne le 01 octobre 2015, consulté le 23 octobre 2020. URL http://journals.openedition.org/temoigner/1224 ; DOI : https://doi.org/10.4000/temoigner.1224 


\section{LE MUSÉE JUIF ET LE CENTRE POUR LA TOLÉRANCE DE MOSCOU}

VISITE Créé à l'initiative de Dov-Ber-Pinhos Berez Lazar, Grand Rabbin de Russie depuis 1999, le musée juif de Moscou a ouvert ses portes en novembre 2012. L'inauguration s'est déroulée en présence du président d'Israël Shimon Pérès et du ministre des Affaires étrangères de Russie, Serguei Lavrov.

e musée est le fruit de financements privés, de subventions du gouvernement russe et de la Fédération des communautés juives de Russie, que qui avait prospéré sur les marches orientales de l'empire russe. Le président Poutine a versé un mois de son salaire au fonds du musée

Alors que Moscou a été défigurée par des constructions boursouflées et monumentales, le bâtiment du musée (ancien dépôt d'autobus de la rue Bakhmetev) a sauvegardé sa silhouette. Conçu par Konstantin Melnikov et Vladimir Choukhov en 1926-1927, ce bijou du constructivisme placé au cœur du vieux quartier juif de Marina Rochtcha, avait été porté, en 1997, sur la liste de monuments à privatiser et cédé par la ville au Centre communautaire, dont le président était alors lentre com lopinion publique et des professionnels a pention de Popinion publique et des professionnels a permis de iter les dégât

L'espace d'exposition est organisé selon un schéma circulaire. Un centre à double noyau est entouré de parois formant deux cercles concentriques : le «grand cosmosjuir»-berceau dujudaisme, anciens Hebreux

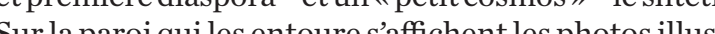
tros lillusUne deuxième paroi, plus excentrée, crée avec Une deuxiène paroi, plus excentrée, crée avec la premiere un courlour où sorganise le parcours chrocologique. Au fond, de grandes salles sont réserveés hac a cótés période historique. Des parapets fixes desc circonstancies. plus circonstanciées.
Le musée affiche l'ambition de dépasser le côté muséal traditionnel et de susciter l'intérêt par sa

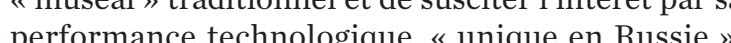
perous dit-on. Il se veut un lieu de distraction capie », d'attirer los jeunes. été confiée à l'agence américaine de Buff eté conflée à lagence américaine de Ralf Appelbaum, à Washingur so visiter do à Washington. Le visteur ne rencontre sur son paret incurvés t tentôt interactifs, tat tot moyens, plats pannés de légndes écrites ou sonores. $3 \mathrm{D}$, accompayx sont réć

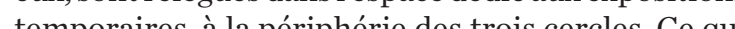
temporaires, à la périphérie des trois cercles. Ce qui ce sontles paroles du poète Iossif Brodski, suréć desus a la boutique du musée : Les objes audessus... de la boutique du musée : « Les objets que nous produisons de nos mains en disent plus long sur nous que nos confessions. » Des " confessions », on n'en trouvera dailleurs pas davantage ici que de "produits de nos mains $x$. Lepeuple des artisans et peuple duLivre nesontplus lls peuple virtuel.

L'espace « shtetl » est à cet égard exemplaire : les visiteurs y sont invités a se déguiser en costumes traditionnels juifs virtuels, à toucher des harengs virtuels sü̈eux dans des figures en plâtre, comme celle qui est aiessise dans des figures en platre, conné celle qui est assise sur un bance dynagogue, plongee dans la lecplicable oubli, cevieux Juifen plâtre n'est par in inexplicableoubl, ce vieux Juifenplatren nt pas interactif et ne se balance pas). La meilleure trouvaille, c'est san lavitesse voulue sous la pression d'une baguette électronique.

Comment l'histoire des Juifs de l'empire (1775-1917), puis de l'URSS (1918-1991) est-elle conçue? Il fallait trourere est-elle conce? Il fallait cepres

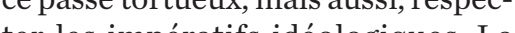
ter les inperatis idén première directrice du musée et du le musé n’avait pas vocatioñ gense navait pas (sic): « Nous n'allons poviettique» larmes sur nous-mêmes. Il ne s'agit larmes sur nous-nênes. Ih ne sagit pas dexposer nos offenses, ils sagite commentl'histoire de notre pays nous cón façonńs et ço

Leparcours chronologique souvre sur une carte de la « zone de résidence » et du Royaume de Pologne

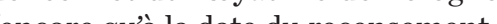

(elui-ci ne s'appelait plus que "pays de la vistule »), qui indique poŕé iècle est tìs chic sur la provenance polon Xine maje est tìs claise se des pure partie de Ju Ju dela delas silence le fait que les Jufs pol quent pas de la sécuć la la Lasection

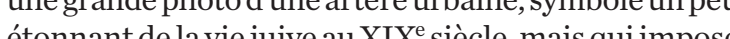

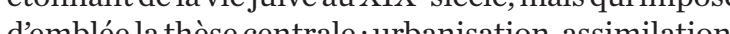

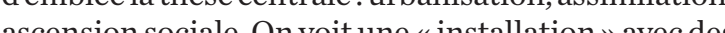
ascension sociale One sées inc cafe ci, Simon Doubnov, Sho ces in juive Sur a pas person juive. Sur la paroi, une "Chronique des evénements juives), presse juive, réveil cultulah (les Lunières juives), presse juive, reverl culturel, assinilation, pogrons, enigition, conr. devant dinfore entisémite Les Protocoles des Sages de Sion, a y trouver $L$ e place), une heur «Destins juifs » permet d'entrer dans le temps indivi- uel et de suivre, à l'aide d'écouteurs et d'un petitécran, des biographies d'hommes remarquables.

a section suivante est intitulée «La Léch défiles p'réce allemande, les scènes des ŕvolutions de févriet doctobre1917, laguerrecivile tên cot "'plus créms que les pogrons de Khmehnitski», (mais lantisénitisme programmatique des Blancs est gorésid. On aborde les questions difficiles : la «zone de droits civiques. Ils doivent tout à la révolution, et les stescos jusqu' in

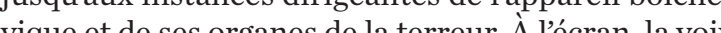
d'un prin spécialiste doutre-Atlantique explique quavec

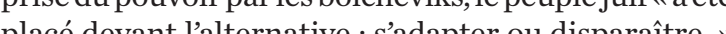
On se dent la te peuples de l'UBSS. Tout come d'autres les Juifs, nous dit... Tout con éta dautres ćs d'ants,

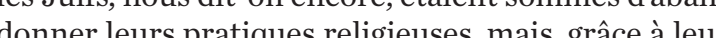
donner leurs pratiques religieuses, mais, grâce à leur niveau déducation supérieur, lls ont vite réussi leur élites intectuel hot et professionnelles. 
- On commence à saisir la fonction du grand écran ont est dotée chaque section : il doit inscrire coûte que commer los conflits, tuer dans l'œuf toute vellét

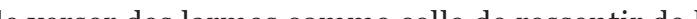
der

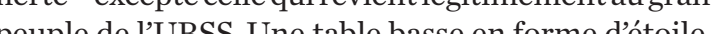
cinq branches incarne cette jeune nation sovietique ; lor sa surface interactive évoluent, comme perdus dan espace, desportraits de persontalités juives: une foi ala portée du visiteur, on les effeure e deploie dans sa courte biographie.

On approche de la salle consacrée à la Grande

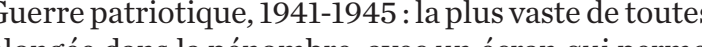
plongée dans la pén. plofe de loffen louest de IURS, abandonnés par larmée sovietique écurer équences prises à Babi Yar. De longs moments terces approche de Stalingrad, des vies connues, des bruit

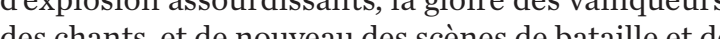

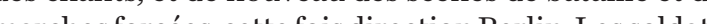
marches forcées, cette fois direction Bélin. Les soldat traversent les villages brulés de la Biélorussie et de prituanie. Et deja viennent les photos canoniques de la prise de Puis ce sont les lendemains de la victo po revient sur le lieu du masace on revient sur le lieu du massacre de Babi Yar, où les centaines de milliers de victimes n'ont pas eu droit au

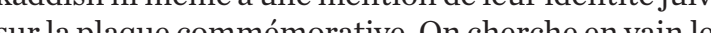
sur la plaque commémorative. On cherche en vain les suifs Qun les Juifs. Quelque chose, quelqu'un, manque dans ce uban ' mages : mais oui, C'est Stalne. Le grand Sta-

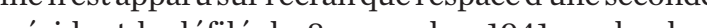
preude. Le Généralissime qui a porté le grand peuce soviéticue à la victoin qui a pouvé le ve grand peuple de Juifs soviétiques et de quelques-uns d'au millions de Juifs soviétiques et de quelques-uns d’autres pays (quand il ne les a pas livés à la Gestapo ou ne les a pas Il ne s'ait plus d'arrondir les angles ; c'est une chape 1 ne s'agit plus darrondir les angles; c'est une chape Stalince qui tombe sur celui qui, dés la victoire de stalngra, fut lordonateun et de la propagande chauvine grand-russienne.

Stalye à loent,

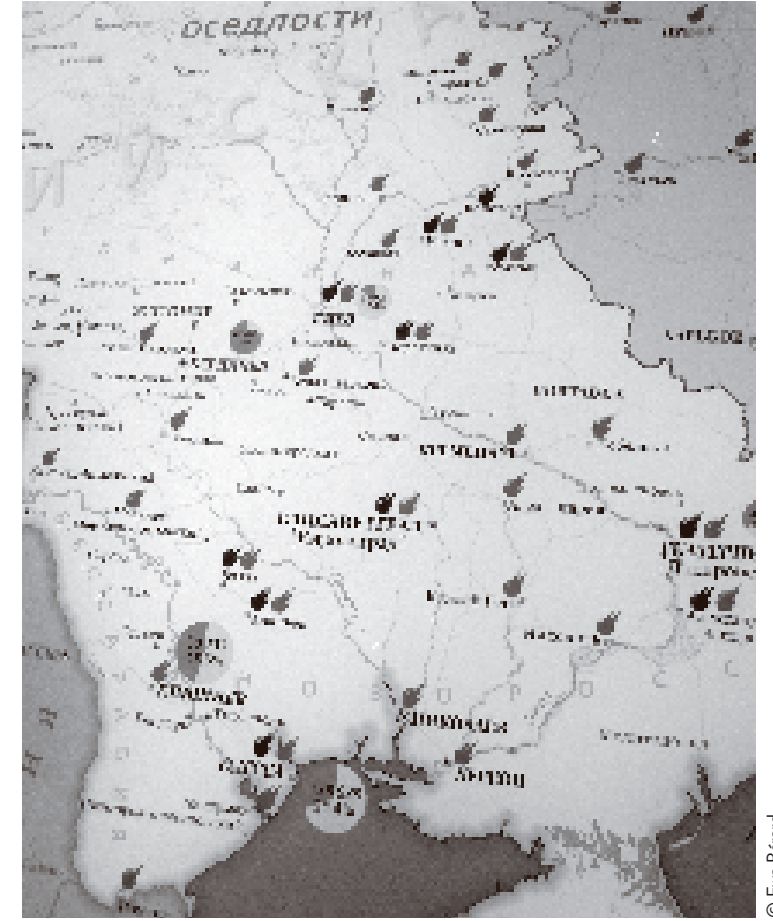
- Carte des pogroms ouvrant le parcours chronologique,
indiquant le pourcentage des Juifs dans la population et
l'intensité des pogroms.

la guerre : le roman Vie et destin de Vassili Grossman. Les intérêts des deux parties prenantes du musée se sont conjugués dans cette stratégie d'évitement : la doxa poutinienne, qui exclut d'avance tout ce qui pourrait ternir linage de la Grande Guerre patriotique, $a$ fortiori une mise en parallèle des régimes stalinien et hitlerien (axe central du roman de Grossman), et Habad, qui n'a aucune raison de remettre a l'honneur la culture des Juifs soviétiques laïcs, ci-devant com-

Décontenancé par cette histoire tronquée, on se tourne vers les panneaux explicatifs. Oui, le pacte Ribbentrop-Molotov est évoqué, ainsi que ses conséquences pour les Juifs polonais. Oui, on parle des ghetlos et de la collaboration de la population locale avec loccupant. Le Comité antifasciste juif (CAJ), créé en 1942, est présent, mais sans que soit mentionnée sa vocation mondiale nil assassinat, sur l'ordre de Staline, juifs polonais. Du célèbre Livre noir, le visiteur peut voir la couverture avec les noms de ses deux rédacteurs, Ehrenbourg et Grossman, mais il ne connaîtra ni so artistes artistes, éc ivains a proposé au CAJ de publier un livre sur le sort des du musée affis publication publicatio tou tle me même que tout le matériel d imprimerie de la maison

celle d'antisémitisme ui de l'Allemagne hitlérienn des annés tilte est cantonnée dans une petite sal À l'entrée, un par troubles : années 1940-1950 truse, cette expression désigne traditiotorographie vace, cen vacance du pouvoir auXVII siècle, quand le pays aétè pro forcéne Sure, se browille.

Sur lem Cin photo Cinq piliers muns chacun d'un écran présentent au visec une légende sur son ćlèbre rapport d'o Jan, avec une légende sur son célebre rapport d août 1946 qui a lancé la r canpas contres hoto de Solomon Mikhoels, presilent du CAJ, photo de Solomon Mikhoels, président du CAJ, avec lééburériode a d'antisé ent jét de répression

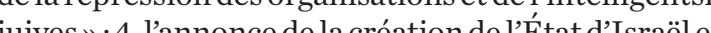

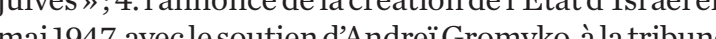
des Nation Unies . fierté que cet événement « áveillé dans les cent de no » que cet événemét « a eveille dans les cœurs

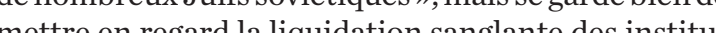
ions et d'un journa tions et des leaders juifs, 5 . la manchette d un journ blouses blanches . Sur ces cinq piliers, le nom de Staline appart une seute fois ; sa photo, jam ine apparait une seule fois, sa photo, jamais.

A mesure que lon se rapproche du jour présent, Juifs soviétiques des années 1960, 1970, 1980, aur des Juins sovíchues des ancés 1960, 1970, 1980, aucune cartedelemigration, aucunemention de laproduction demment, de la répression et de l'antisémitisme d’État
Deux écrans seulement : le premier, en 3D, présente n appartement moscovite où deux jeunes gens bien êr personne échangent des blagues juives tandis qu'une jeune femme sort d'un carton des cadeaux envoyés par les parents installés aux USA. Sur le deuème, un groupe de Juifs se réunit clandestinement dans une forêt pour encourager, dans l'esprit comes activités sportives de leurs

generation grandit, saine de corps et d'esprit.

La visite est terminée - ou presque. Il reste explique-t-on, se déro la De quoi y discute-ton? Les questions d'o duatié préoccupentla sociétérusse tellesqul'titude qu les perpentes a d'orientation es migrants asitiqus, sont-elles souleves? Non, mes migrants asiatiques, y sont-elles soulevees ? Non, mes propose deux brochures. On y apprend que lestages propose doux bróches. On y apprend que les stages étris es buts à pindre une sur ests at indre « sur le plan personnel» figurent: et créative dans la vie de la société et de l'śtat. Motivation et orien lation dur les valeurs, fondés sur idées du patritisme de l'omour tu Patrie et sur la recon de lanour et du respect pour

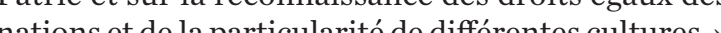
Est-ce donc ici, dans cette éducation à la utolérance" qui n' qui n'est en fait que le condśconnement à lingènierie pour com à la discipline dEtat, qu'on tioure la lo toire des Juifs soviétiques? Version qui semble avois on ànevir pour une deuxième visite. Fascinés parbres formance des gadgets high-tech, les mêmes interturnan qún gat igh-tech, les mêmes intellectuels qui dénoncent, indignes, le retour des pratiques

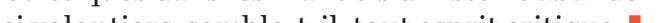
Ewa Bérard, octobre 2013

La version élargie de ce texte est à paraître dans la
Revue des Etudes Slaves.

Lauteure remercie les collaborateurs du musée quil ui ont gênéreusement consacré leur temps et ont vaillamment répondu à ses questions.
Il sest tevident tue eles opinions exprimées dans cet article nengagent
quélle-même.

\section{Ewa Bérard, chercheur au CNRS, a publié, entre autres, La Vie} tumultueuse d'llya Ehrenbourg. Juif, Russe et Soviétique, Paris, Ramsay,
1991 (traduction polonaise, Varsovie, 2002, traduction russe, mise à jour, 